Research

Article

\title{
On Schedulability Analysis of AADL Architecture with Storage Resource Constraint
}

\author{
Yin Lu (陆寅 $)^{1}$, Shudong Qin (秦树东 $)^{2}$, Leqi Xi (习乐琪 $)^{1}$, Yunwei Dong \\ (董云卫 $)^{1}$ \\ ${ }^{1}$ (School of Computer Science, Northwest Polytechnical University, Xi' an 710072, China) \\ 2 (School of Software, Northwest Polytechnical University, Xi' an 710072, China) \\ Corresponding author: Yunwei Dong, yunweidong@nwpu.edu.cn
}

\begin{abstract}
Embedded systems have been widely applied in real-time automatic control systems, and most of these systems are safety-critical, for example, the engine control systems in an automobile and the avionics in an airplane. It is very important to verify the schedulability of such a real-time embedded system in its early design stages, so as to avoid unexpected loss for the debugging of architecture design problems. However, it has been proved to be a tough challenge to evaluate the schedulability of a Preemptive-Scheduling Real-Time (PSRT) system, especially when the constraints of system resources are taken into consideration. The cache memory built inside the processor is an exclusive-accessing resource shared by all the tasks deployed on the processor. In addition, the Cache-Related Preemption Delay (CRPD) caused by preemptive task scheduling will bring extra time to the execution time for all the tasks. Thus, the CRPD should be taken into consideration when the Worst-Case Execution Time (WCET) of tasks is estimated in a real-time system. A model-based evaluation and verification method of architecture schedulability, which is designed for priority-based PSRT systems, is proposed in this study to make cache resource constrained and CRPD related schedulability evaluation based on the AADL system architecture model. In the first step, the study enhances the property set of AADL storage elements to make it compatible with cache memory properties in a system architecture model. Secondly, the study proposes a set of algorithms to estimate the CRPDs of a task before it is completed; run system schedule simulation and construct the schedule sequence with the constraint of cache resources and CRPDs involved; and make WCET estimation of the tasks in such a CRPD considered, preemptive-scheduling execution sequence. Finally, the methods mentioned above are implemented within a prototype software toolkit, which is designed to make evaluation and verification of system schedulability within CRPD constraints. The toolkit is tested with a use case of aircraft airborne open-architecture intelligent information system. The result shows that, compared with the schedule sequence constructed without cache memory resource constraints, the WCET estimated for most tasks is extended, and the sequence order is changed. In some extreme cases, when CRPD is taken into consideration, some tasks are evaluated to be incompletable. The test shows that the method and algorithms proposed in this study are feasible.
\end{abstract}

This is the English version of the Chinese article “面向 AADL 模型的存储资源约束可调度性分析. 软件学报, 2021, 32(6): 1663-1681. doi: 10.13328/j.cnki.jos.006243”.

Funding items: National Natural Science Foundation of China (61772423)

Received 2020-08-31; Revised 2020-10-26; Accepted 2020-12-19; IJSI published online 2021-12-23 
Keywords AADL; complex embedded system; cache-related preemption delay; resource constraint schedulability

Citation Lu Y, Qin SD, Xi LQ, Dong YW. On schedulability analysis of AADL architecture with storage resource constraint, International Journal of Software and Informatics, 2021, 11(4): 429-452. http://www.ijsi.org/1673-7288/255.htm

As widely used in safety-critical fields such as aerospace, automotive electronics, and telemedicine, complex real-time embedded systems have high requirements for system reliability, safety, and real-time performance. Particularly, they have strict time constraints in terms of realtime performance. They are required to complete before the deadline, which is a kind of execution time constraint. Therefore, we have to analyze the real-time performance of such systems from the perspective of software architecture at the early stage of design, and use the analysis results to guide system design and implementation. This is conducive to the real-time performance of systems.

The conventional schedulability determination of embedded systems uses the Worst-Case Execution Time (WCET) of each task in systems and specific scheduling algorithms to determine whether the WCET of each task can meet its deadline. WCET can be indicated as an upper bound on the execution time of a task on a processor ${ }^{[1]}$. Therefore, we need to estimate the WCET of tasks to analyze the real-time performance at the design stage of system models. However, WCET is generally dependent on multiple resource constraints of a computing platform. Currently, most of the static and dynamic calculations for WCET estimation only take the constraint of processors into consideration ${ }^{[2]}$. In static calculation, the maximum possible execution path of a program is analyzed according to the control flow graph of the task, to obtain the WCET of the task ${ }^{[2]}$. In dynamic calculation, a program is run multiple times to test its execution time in each round of running, and the maximum execution time in the test results is taken as the WCET of this task. However, this method calculates WCET in software oriented program code and cannot analyze and evaluate the schedulability of software during requirement planning and model design.

As embedded systems are increasingly complicated, scholars have adopted a stepwise refinement and hierarchical modeling process in software development, to guarantee that the non-functional properties of software are meet in implementation. Non-functional properties are critical to the quality of software, and guarantee of thier implementation is of great challenge in software development. Recently, the Architecture Analysis and Design Language $(\mathrm{AADL})^{[3,4]}$ has been proposed to solve the difficulties in designing and analyzing the nonfunctional properties of complex systems. AADL supports both textual and graphical software architecture design, and adopts a top-down system engineering idea, which can greatly reduce development cycles and costs. The schedulability verification based on AADL models is an important research direction in the non-functional property design and verification with AADL. Generally, the schedulability verification of AADL models is based on specific scheduling algorithms, such as Rate Monotonic Schedule (RMS), Earliest Deadline First (EDF), and Fixed Priority Scheduling (FPS). In general, the priority of a task changes dynamically during its execution period. Then, processors dynamically schedule tasks according to their priorities in the scheduling process. EDF is a typical dynamic priority scheduling strategy that takes the task whose deadline is closer to execution. With the help of EDF, the schedulability verification method based on processor utilization is also proposed. Specifically, if the sum of processor utilization rate of all tasks in a task set is less than 1, the task set will be regarded as schedulable with the EDF strategy ${ }^{[5]}$.

The schedulability analysis of AADL models based on a timed automata model is becoming an approach to study the real-time performance of complex systems. It uses a timed automata 
model to simulate the dynamic behavior of each model component in the system, and then analyzes the schedulability of the system model in design stage according to the real-time requirements and behavior specifications of it. This can guide the classification of functions and decomposition of performance indexes of complex systems in their early design stage. Some research institutions have made fruitful attempts. For example, scholars from the University of Pennsylvania developed a schedulability analysis tool, verification execution, and rewrite system for ACSR (VERSA). It automatically transforms AADL models to ACSR models and builds timed automata models of threads in toolkits. It models processors and accesses as resources while data and event ports as communication channels ${ }^{[6]}$. Scholars from the University of Illinois Urbana-Champaign developed a language and tool, Real-Time Maude, which supports formal description, simulation, and analysis of real-time systems ${ }^{[7,8]}$. It conducts formal modeling for the system and then performs analysis and simulation to analyze the schedulability of a system. In addition, the toolkit aadl2sync supports formal verification of the schedulability of AADL models. However, aadl2sync does not support AADL modeling and has deficiencies during transformation. For example, it converts asynchronous concepts into synchronous ones, resulting in the incomplete conversion of AADL models ${ }^{[9]}$. Cheddar, an open-source real-time scheduling computing framework developed by Brest University, supports a variety of scheduling strategies. It also supports the analysis of AADL port delays, shared resources, and message queues $^{[10]}$. Li et al. proposed transformation rules between the AADL behavior model and the timed automata model of UPPAAL based on the semantics of the AADL behavior model by combining the Behavior Annex (BA) in AADL. In addition, they proved the correctness and validity of the conversion and verified the system schedulability by simulation in UPPAAL ${ }^{[11]}$. However, these tools only consider the schedulability analysis under the single resource constraint of processors. In the early design stage of a real-time system, the system schedulability with multiple resource configuation strategies should be analyzed comprehensively.

Complex real-time embedded systems usually adopt a preemptive scheduling strategy to guarantee a timely response to critical tasks. Preemption makes the execution time of tasks uncertain, which increases the computational cost. For example, preemption may lead to cache interference, and cache blocks of the preempted task are replaced out of the cache. When the preempted task is re-executed, these replaced cache blocks have to be reloaded. The repeated loading time is called Cache-Related Preemption Delay (CRPD). Some scholars in China proposed calculation methods of CRPD, such as the UCB-ECB union (Useful Cache Block-Evicting Cache Block union) algorithm. Meanwhile, they also provided the comparison results between this method and other algorithms ${ }^{[12]}$. Hai Nam Tran et al. proposed a CRPD calculation method for analyzing the path of preempting tasks by constructing the Control Flow Graph (CFG) of tasks. This method defines UCBs and ECBs for each node in CFG, and calculates the CRPD of tasks using UCB-Union ${ }^{[13]}$. It provides a good idea to calculate the cost caused by the preemption behavior of executable components during dynamic operation. Meanwhile, Hai Nam Tran et al. proposed an AADL cache modeling method. They make use of the subprogram component in AADL to build models for CFG of programs and employ Ada to extend the Cheddar tool so that it can calculate CRPD according to the CFG of programs ${ }^{[17]}$. In addition, to guarantee the isolation between tasks in a complex embedded system and reduce error propagation and interference, scholars usually adopt partitioned scheduling strategies to isolate applications at different security levels, which run on the same computing platform. The reliability and security of a whole system can be improved by ensuring the real-time performance of each partitioned task. However, at the architectural design level, AADL cannot describe a specific CFG for program execution, so the preemption delay for component interaction cannot be calculated with the existing modeling and analysis methods of AADL. Therefore, to overcome 
the problem, that is there is no clearly information of control flow and dataflow in the model because of there is no implementation code of the program, in the calculation of CRPD, this paper proposes a CRPD calculation method based on preemption sequences. This method analyzes the schedulability of AADL scheduling models under the CRPD constraint in a generalized way without requiring the specific code implementation of model components.

Aiming at complex embedded systems, this paper studies the schedulability of AADL models under the constraint of CRPD in the early design stage of system architecture. By extending the modeling technology of AADL storage resources, we develop an intra-partition architecture model containing basic scheduling elements and an architecture model of a partitioned system satisfying the ARINC653 standard $^{[15]}$ to study the schedulability of AADL architecture models under the constraint of CRPD. In addition, we propose the calculation method of CRPD based on preemption sequences and the calculation method of WCET under the constraint of CRPD. Our method is not concerned with the specific code implementation of the AADL thread component and is more suitable for real-time analysis scenarios based on AADL architecture models in the early development stage of software. In the design stage of embedded software models, our method provides theoretical guidance on the schedulability determination with the CRPD constraint. It can avoid repeated development induced by poor real-time performance due to a high preemption cost between tasks in the development process of embedded software.

Section 1 extends the modeling capacity of AADL, aiming at the deficiencies of AADL in cache resource modeling. Section 2 introduces cache and the calculation method of CRPD. Given scheduling strategies, the cache-related delays caused by the operation of each component in the system architecture are analyzed, and a calculation method of estimating delay based on preemption sequences is proposed. In addition, a schedulability determination method and a scheduling strategy are proposed for task sets with the storage resource constraint. In Section 3, the prototype architecture of a schedulability analysis tool is designed for AADL architecture, and schedulability analysis experiments are conducted for an open intelligent airborne information system under the cache constraint. At last, the work in this paper is summarized, and future research is forecasted.

\section{Modeling of AADL Cache Resource}

AADL is a modeling language for designing and analyzing architecture models of complex real-time embedded systems and for analyzing non-functional properties. It provides the Memory component to model system storage resources and describes the properties of storage resources used by software components through a binding mechanism. AADL models support hierachical storage modeling ${ }^{[13]}$ and use the Memory components in a recursive nested structure to describe the hierachical structure of Memory. AADL models map storage resources from the perspective of software and hardware by bounding software to the Processor component and divide the memory into segments. However, due to the lack of properties describing the dynamic execution of processes in AADL, it is impossible to further describe the segmentation properties of the Memory component used by processes and threads of AADL components. Thus, we need to model Cache with the Memory component as a subcomponent of the Processor and portray the cache structures such as one-level cache and two-level cache through the internal structure of Memory in AADL. Among them, two-level cache modeling can be used for multicore systems ${ }^{[16]}$.

To analyze the CRPD of components at the architecture level with the AADL model, this paper extends the properties of the Memory component in AADL and subdivides the hierarchical storage structure in AADL models. In AADL, the Memory component is taken as a subcomponent of the Processor component to model cache, and the Memory properties required 
by competition modeling of scheduling resources are added, as shown in Table 1. CacheSize indicates the size of cache; LineSize describes the capacity of a cache block; CacheMissTime indicates the time delay due to loading a cache block from memory in a cache miss. Generally, the time of loading a cache block from cache is a constant when resource preemption occurs in a specific CPU model. When a cache is not hit, a cache miss occurs. The missed data and the associated blocks should be written from the main memory to the cache, and the data is accessed in the cache. The size of the cache affects the delay of this process and further influences the schedulability of system tasks.

Table 1 Extended properties of memory component in cache modeling

\begin{tabular}{cccc}
\hline Component & Property & Type & Unit \\
\hline Memory & CacheSize & aadlinteger & Byte, KB, MB, GB \\
Memory & LineSize & aadlinteger & Byte, KB, MB, GB \\
Memory & CacheMissTime & aadlinteger & ps, ns, $\mu \mathrm{s}, \mathrm{ms}, \mathrm{s}$ \\
\hline
\end{tabular}

The extended AADL cache model can describe properties related to cache and resource accesses of main Memory components required by the design of the system architecture, such as CacheSize and CacheMissTime. During modeling, the keyword "applies to" can define Line_Size, the property of cache block capacity of the Memory component, and the properties of the Memory component provided by AADL can be used to model the main memory resource. During system architecture design, the AADL component "thread" can define system tasks as well as properties and behaviors related to their scheduling execution, such as scheduling protocols, execution time, task cycles, deadlines, and requirements for accessing storage resources. When systems employ virtual technology to divide and bound partition resources and conduct partitioned scheduling execution, the size of the time slice round robin of the scheduling partition should be defined for each task partition. In the analysis of system schedulability, the memory accessing delay of each component execution in models can be calculated by extracting behavioral properties of partitions and components as well as properties of the memory component in the AADL architecture, as shown in Table 2.

Table 2 Component properties required for CRPD calculation

\begin{tabular}{cc}
\hline Component & Property \\
\hline CacheSize \\
WordSize \\
LineSize \\
CacheMissTime \\
\hline RomBudget \\
RomActual \\
RamBudget \\
RamActual \\
& Source_Data_Size \\
& Source_Code_Size \\
Thread, Thread Group & Source_Heap_Size \\
& Source_Stack_Size \\
& Dispatch_Protocol \\
& ComputeExecutionTime \\
& Period \\
& Deadline \\
\hline
\end{tabular}

When CPU loads resources in a way of extracting the required words of CPU from cache after cache blocks are loaded, cache misses should be analyzed. In this paper, we treat CacheMissTime as a constant for the convenience of analysis. 


\section{Calculation of Cache-Related Delay}

\subsection{Cache-related preemption cost}

In embedded systems, cache resources may cause cache preemption delay and timing exceptions of task execution for real-time scheduling of systems. Timing exceptions often lead to behavior failures but do not affect WCET, so they are not taken into consideration in this paper. In a preemptive system, when preemption occurs between tasks, the content of the preempted task that has been loaded into the cache is replaced out of cache due to cache conflicts. When the preempted task is executed again, the replaced content should be loaded into cache again ${ }^{[17]}$, and the repeated loading time is the cost of task delay caused by cache-related preemption. The root cause of preemption delay lies in the conflicts within mappings of storage resources between tasks.

In a preemptive scheduling environment, the execution efficiency of a task and the real-time performance of a system are often affected by preemptions between tasks. Preemptions of tasks not only bring about Context Switch overHead (CSH) but also cause CRPD. The research results show that during task scheduling, $\mathrm{CSH}$ is about $5 \mu \mathrm{s}-10 \mu \mathrm{s}$, while the computation delay caused by cache-related task preemption may reach $1 \mu \mathrm{s}-10,000 \mu \mathrm{s}$. The computation delay of task preemption depends on the system structure and its cache capacity ${ }^{[18,19]}$. Thus, the real-time property analysis of a system should pay more attention to the delay due to related preemptions caused by the structure of storage resources in the system and its cache capacity.

In the specific hardware and software environment of a system model, the time of CPU loading a cache block from the cache, CacheMissTime, can be regarded as a constant, and it can also be used to indicate the upper bound on the time to load a block of data content from the main memory to cache. Thus, when calculating cache-related preemption cost, we only need to know the number of cache blocks that should be reloaded when the preempted task resumes execution. Then Equation (1) is adopted to calculate the CRPD of a system ${ }^{[13]}$.

$$
C R P D=g \times \text { CacheMissTime }
$$

where (1) $g$ is the number of cache blocks to be loaded when the preempted task resumes execution; (2) CacheMissTime indicates the upper bound on the time required to load a cache block of content from the main memory to cache, and it can be viewed as a constant in specific hardware architecture.

In the hierarchical analysis model of the system architecture, the key to the schedulability of a component is to calculate the CRPD of the component. Since there is no code implementation in architecture models and then no real operation profile to calculate CRPD, how to determine the number of cache blocks that should be loaded when the preempted component resumes execution is a difficulty in the schedulability analysis on system architecture level. If all cache blocks of the preempted task are used to calculate CRPD, CRPD will be overestimated as the replaced cache blocks may not be adopted after the preempted task resumes execution. In this paper, we estimate CRPD based on the numbers of Useful Cache blocks (UCBs) and Evicting Cache Blocks (ECBs). A UCB is the data block that is not replaced out of cache during the program execution, while an $\mathrm{ECB}$ is the data block that needs to be replaced into cache during the execution of the preempting task. Based on the ideas of UCB and ECB, two calculation methods of CRPD, UCB-Union, and ECB-Union, have been proposed ${ }^{[13]}$. We use the UCB-Union method to calculate the overhead of the task $T_{k}$ preempting $T_{i}$, as shown in Equation $(2)^{[14]}$ :

$$
C R P D_{(i, k)}^{\mathrm{UCB}-\text { Union }}=\text { CacheMissTime } \times\left|\left(\bigcup_{\forall k \in \operatorname{bet}(i, k)} U C B s_{i}\right) \cap E C B s_{k}\right|
$$


where (1) $C R P D_{(i, k)}^{\mathrm{ECB}-\text { Union }}$ indicates the CRPD generated by the task $T_{k}$ preempting the task $T_{i}$, which is calculated by the UCB-Union method; (2) $U C B s_{i}$ is the UCB set of the task $T_{i}$; (3) $E C B s_{k}$ is the ECB set of the task $T_{k} ;(4) \operatorname{bet}(i, k)$ is the set of tasks whose priority is at least that of the preempted task $T_{i}$ and smaller than that of the task $T_{k}$ in the scheduled task set.

The ECB-Union method is adopted to calculate the overhead of the task $T_{k}$ preempting $T_{i}$, as shown in Equation (3):

$$
\operatorname{CRPD}_{(i, k)}^{\mathrm{ECB}-\text { Union }}=\text { CacheMissTime } \times \max _{\forall k \in \text { bet }(i, k)}\left\{\left|U C B s_{i} \cap\left(\bigcup_{t \in h p(k) \cup T_{k}} E C B s_{k}\right)\right|\right\}
$$

where (1) $\operatorname{CRPD}_{(i, k)}^{\mathrm{ECB}-\mathrm{Union}}$ indicates the CRPD of the task $T_{k}$ preempting the task $T_{i}$, which is calculated by the ECB-Union method; (2) the meanings of $U C B s_{i}$ and $E C B s_{k}$ are the same as those in Equation (2); (3) $h p(k)$ is the set of tasks whose priorities are greater than that of the task $T_{k}$ in the scheduled task set.

The UCB-Union and ECB-Union methods both overestimate the cache preemption cost, because they add the high-priority tasks not involved in the preemption to the CRPD calculation of the preempted task. Thus, we propose a CRPD calculation method based on preemption sequences aiming at the possible overestimation of cache preemption cost. Our method focuses on how to accurately calculate the CRPD of the preempted task when preemption occurs to determine the schedulability of each component in system architecture.

\subsection{Timed automata model of threads}

To describe the operational behavior of each component in system architecture and simulate the scheduling behavior of component operation, AADL extracts the scheduling and execution behavior of the thread component, the smallest execution unit in the system, as a timed automata model. AADL threads in the state machine include five task states, i.e., ready, running, awaiting resource, awaiting return, and awaiting reentry. For the convenience of indication, the states of awaiting return and awaiting reentry are not considered in this paper as they are not related to the resource constraint. Thus, the simplified execution model of AADL threads can be described by three states, i.e. the ready state, the running state, and the awaiting state, as well as the transition description between states, as shown in Figure 1. In the figure, $c$ is the time accumulation of a thread executing on a processor; $w$ is the time accumulation of threads due to resource blocking; $\partial c$ indicates whether the thread is in the computation state and conducts time accumulation on a processor. When $\partial c=1$, the thread is in the execution state and conducts time accumulation; otherwise, the thread is not in the execution state and does not need to accumulate time. Similarly, $\partial w$ is defined as the time accumulation due to resource blocking. When $\partial w=1$, the thread is in the blocking state and conducts time accumulation; otherwise, it is not in the blocking state, and time is not accumulated. There are four transitions among the three states: (1) the thread acquires the processor time in the ready state and then enters the running state; (2) cache misses in the running state cause processor blocking, and the thread enters the awaiting resource state; (3) in the awaiting resource state, when this thread obtains the resources required by execution, the thread enters the ready state; (4) the thread in the running state enters the ready state after it is preempted by a thread with a higher priority.

Scheduling of complex embedded systems often follows partition scheduling to manage system components and their resources. For example, airborne embedded systems are constructed with the Integrated Modular Avionics (IMA) structure. Embedded systems with this software structure adopt a two-level scheduling model to manage the operation of tasks: inter-partition scheduling and intra-partition scheduling. AADL defines an appendix model 
ARINC653 to support the modeling of partitions. Inter-partition scheduling follows the time slice round robin strategy, so the delay caused by switching between partitions should be considered during system operation. According to the operational semantics of AADL ARINC653, a timed automaton can be used to describe the task scheduling behavior of complex systems with partitions, as shown in Figure 2.

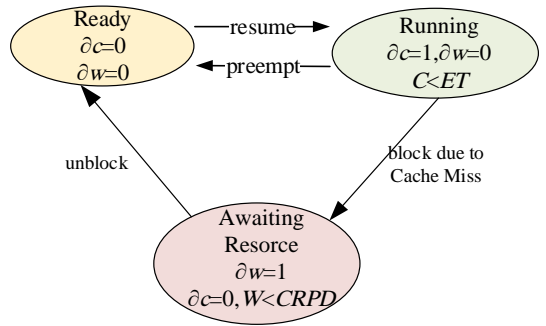

Figure 1 Simplified timed automata model of AADL thread component

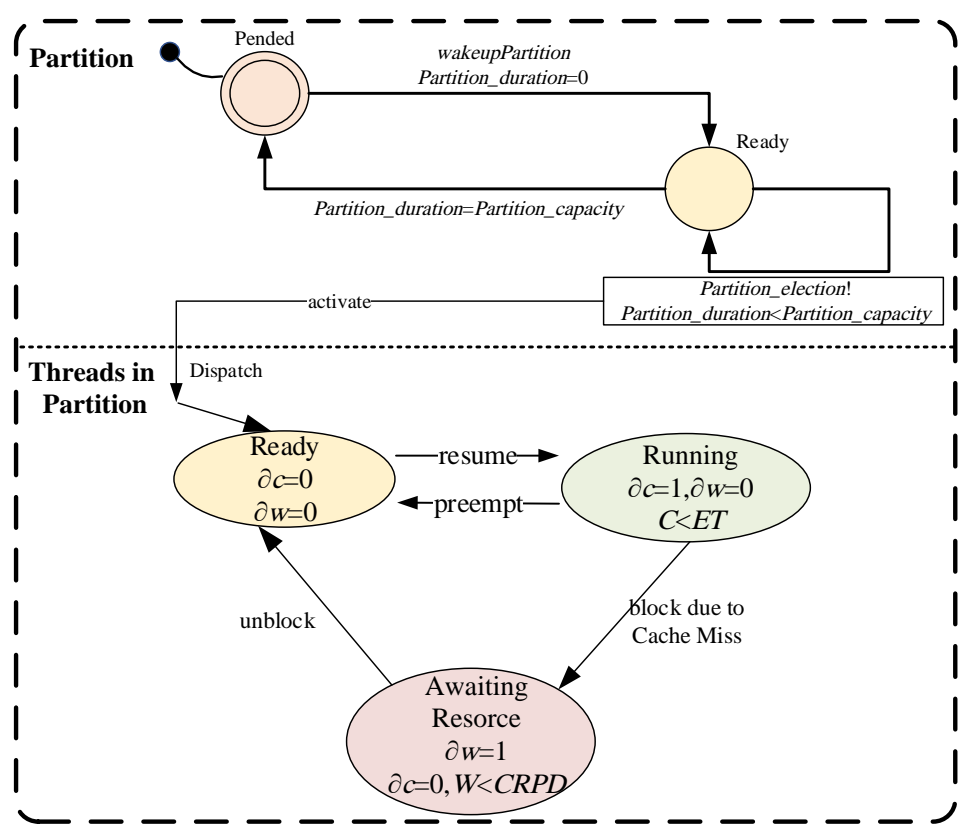

Figure 2 Timed automata model of ARINC653 partitioned system

The system allocates time slices of the processor to each partition according to the partition configuration table. Based on the time slices obtained by each partition, the intra-partition scheduling strategy dispatches the threads in the partition according to the scheduling policy specified by the partition. In the partition scheduling model shown in Figure 2, the state machine of each system partition includes the ready state and the pended state. The pended state of a partition indicates that the partition is in the blocking state and does not receive the time slice information of the processor. When wakeupPartition activates the partition, the time that the partition has been executed is recorded as Partition_duration $=0$, and the partition enters the ready state. The ready state indicates that the partition is assigned time slices of the processor and activates threads in it, and the thread with the highest priority in the partition is selected for execution. When the actual execution time of the partition is equal to the time slices 
(Partition_capacity) that it acquired, the computation time of this partition is exhausted, and all tasks in the partition enter the blocking state.

\subsection{Calculation of preemption sequences}

The execution cycle varies in different components of the system architecture. It is necessary to define the operation benchmark of a task set in a superperiod to calculate the preemption cost of each component accurately and consistently. The superperiod of a task set is the lowest common multiple of the execution cycles of components in the task set. For a more accurate estimation of the time overhead of task preemption of a task set within a superperiod, it is necessary to identify all the preemptions between tasks within the superperiod, that is, to find the preemption sequence. To calculate preemption sequences precisely, we divide the preemption method into explicit and implicit preemptions. Explicit preemptions refer to the task scheduling without cache block switching caused by access conflict of memory resources when preemptions occur. In this case, for the preemptions generated by tasks under a specific scheduling strategy, there is no need to consider the CRPD cost at each task preemption point. Implicit preemptions refer to the task scheduling in which cache blocks are switched due to access conflicts of memory resources during the task execution because of limited cache resources when preemptions occur. In this case, when tasks are preempted under a specific scheduling strategy, it is necessary to consider the CRPD cost at each preemption point. For example, there are three thread components in a system, and the corresponding set of execution tasks is $\left\{T_{1}, T_{2}, T_{3}\right\}$. The properties of the scheduling behavior of each task are shown in Table 3.

Table 3 Task properties in scheduling simulation

\begin{tabular}{cccccc}
\hline Task & Priority & $\varphi$ (dispatched phase) & ET $(\mathrm{ms})$ & Period $(\mathrm{ms})$ & DeadLine $(\mathrm{ms})$ \\
\hline$T_{3}$ & 3 & 30 & 10 & 50 & 50 \\
$T_{2}$ & 2 & 10 & 10 & 50 & 50 \\
$T_{1}$ & 1 & 0 & 17 & 50 & 50 \\
\hline
\end{tabular}

Figure 3(a) shows the scheduling sequence of this task set within two superperiods, and the fixed priority method is taken as the scheduling algorithm. In the scheduling process, task $T_{1}$ is explicitly preempted by task $T_{2}$ at $10 \mathrm{~ms}$ and $60 \mathrm{~ms}$, and the scheduling of tasks does not consider the effect of the CRPD cost on scheduling.

However, when the CRPD cost is considered and the CRPD cost at each preemption point during the task scheduling is high, the WCET of the whole task set calculated based on explicit preemption points may be too low. In the scheduling process shown in Figure 3(b), assuming the CRPD cost generated by task $T_{2}$ preempting task $T_{1}$ at $10 \mathrm{~ms}$ is $6 \mathrm{~ms}$, the WCET of task $T_{1}$ based on CRPD is $23 \mathrm{~ms}$; therefore, the preemption of $T_{3}$ to $T_{1}$ will be additionally increased. When $T_{1}$ is re-preempted by $T_{3}$, if the preemption cost of $T_{3}$ at the preemption points $30 \mathrm{~ms}$ and $80 \mathrm{~ms}$ is bigger than the preemption cost of $T_{2}$ to $T_{1}$ at $10 \mathrm{~ms}$ and $60 \mathrm{~ms}$, which is $6 \mathrm{~ms}$, the scheduling delay of task $T_{1}$ will be underestimated if the scheduling delay is still calculated by the scheduling method shown in Figure 3(a). As such, the CRPD at these implicit preemption points should be considered when the WCET of the task set is calculated.

Given a scheduling policy, a preemption sequence indicates the execution sequence of highpriority tasks in a task set between the time when a task is preempted and the time when the task is re-executed. preemptionSequence $\left(T_{i}\right)$ indicates the execution sequence of high-priority tasks in the period from task $T_{i}$ being preempted to it being executed, and preemptionSequence $\left(T_{i}\right)=$ $\left(T_{x}, T_{y}, T_{z}\right)$ represents that the execution sequence of the task set in the period from the task $T_{i}$ being preempted by the task $T_{x}$ to the task $T_{i}$ being executed is $T_{x} \rightarrow T_{y} \rightarrow T_{z}$. Accordingly, before calculating the CRPD of each execution task, we should confirm preemption sequences of that task and calculate the CRPD of each possible preemption in the sequence. Then the 
CRPD results of all preemptions are added to obtain the CRPD of this task.

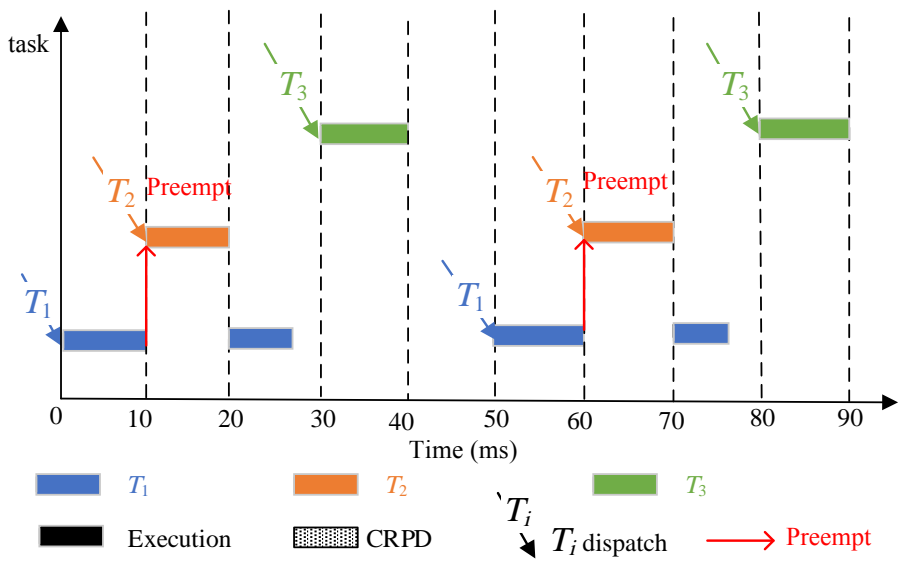

(a) Explicit preemption scheduling sequence

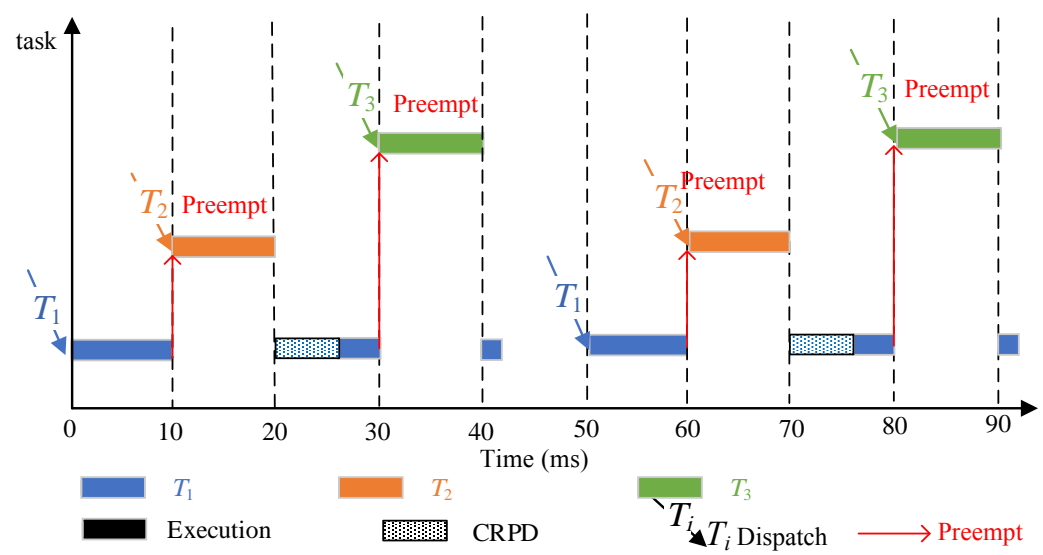

(b) Implicit preemption scheduling sequence

Figure 3 Comparation between task scheduling sequences in different contexts

For scheduling strategies, RMS and EDF, preemption within a superperiod can only occur at the moment when a high-priority task arrives. We call these points execution breakpoints of tasks, and preemption points of tasks are a special type of execution breakpoints. Thus, when analyzing the schedulability of tasks, we should first calculate the execution breakpoints of all tasks within a superperiod. It is assumed that there is a task set Taskset $=\left\{K_{1}, K_{2}, K_{3}\right\}$, where the cycle of $K_{1}$ is $2 \mathrm{~ms}$, that of $K_{2} 3 \mathrm{~ms}$, and that of $K_{3} 6 \mathrm{~ms}$. Then the superperiod of this task set is the lowest common multiple of the cycles of the three tasks, i.e. $6 \mathrm{~ms}$. The execution breakpoints are $(0 \mathrm{~ms}, 2 \mathrm{~ms}, 3 \mathrm{~ms}, 4 \mathrm{~ms}, 6 \mathrm{~ms}, 8 \mathrm{~ms}, 9 \mathrm{~ms}, 10 \mathrm{~ms})$. In the case of partition scheduling, switching points between time slices in partition scheduling are also regarded as execution breakpoints of tasks (as shown in Figure 4).

Algorithm 1 provides the computational process for all execution breakpoints in a task set. There are a Do-while loop and a for loop ( $m$ partitions and $n$ tasks in the task set). The Do-while loop is executed for $m$ times, and the for loop is executed for $n$ times. There is no nesting relationship between loops, and $m$ is smaller than or equal to $n$, so the time complexity 
of Algorithm 1 is $\mathrm{O}(n)$.

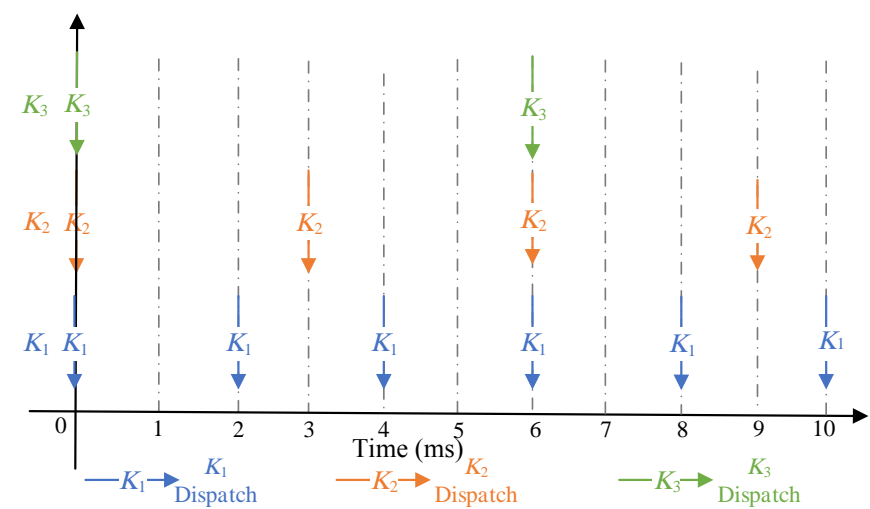

Figure 4 Dispatch of tasks in the task set within a superperiod

Under the scheduling strategies RMS and EDF, the preemption of a high-priority task to a low-priority task must occur at the moment when the high-priority task arrives. Then, to determine whether an execution breakpoint is a preemption point, we can judge whether the remaining execution time of the current task is zero when the execution breakpoint arrives and whether there is a high-priority task arriving. Therefore, after calculating the execution breakpoint sequence of a task, we should also calculate the remaining execution time of the task to determine whether the execution points are preemption points. With the execution sequence of tasks $T_{1}$ and $T_{2}$ shown in Figure 5 as an example, the execution breakpoint sequence between $K \mathrm{~ms}$ and $K+5 \mathrm{~ms}$ is $\{K \mathrm{~ms}, K+1 \mathrm{~ms}, K+2 \mathrm{~ms}, K+4 \mathrm{~ms}\}$. Assuming the current execution breakpoint is $K+1 \mathrm{~ms}$, the previous execution breakpoint is $K \mathrm{~ms}$. Task $T_{1}$ is dispatched at $K$ $\mathrm{ms}$, and the execution time required for $T_{1}$ is $1.5 \mathrm{~ms}$. Thus, at the current execution breakpoint, task $T_{1}$ has not been completed. At the current execution breakpoint $K+1 \mathrm{~ms}$, task $T_{2}$ arrives; the remaining execution time of $T_{1}$ is $0.5 \mathrm{~ms}$, and $T_{1}$ is preempted, so the current execution breakpoint is a preemption breakpoint. Thus, after calculating all the preemption breakpoints during the execution of a task, we can get the preemption sequence of this task in the scheduling process.

Algorithm 2 provides the process of generating a preemption sequence by determining preemption points, and it contains a Do-while loop, and the loop variable is the execution breakpoint sequence. The size of this sequence is related to each task cycle, which is provided by designers, so the time complexity of Algorithm 2 cannot be estimated based on the number $n$ of tasks. Assuming that there are $k$ elements in the execution breakpoint sequence, the time complexity of the algorithm is $\mathrm{O}(k)$.

In Algorithm 2, if a task needs to get processor resources, it is in a ready state and in a ready queue. If the task has the highest priority in the ready queue, it gets the processing time. A task execution breakpoint indicates that a new task is coming. If the new task is added to the set of tasks in the ready state, the scheduler selects the task with the highest priority in the set and changes its state to running. When the executed task is preempted, the task state is switched to ready and the task is added to the ready queue. When the task being executed is completed or blocked, the task enters the awaiting state. Thus, a task enters the awaiting state when it finishes execution in one cycle and enters the ready state when it is dispatched in the next cycle. 


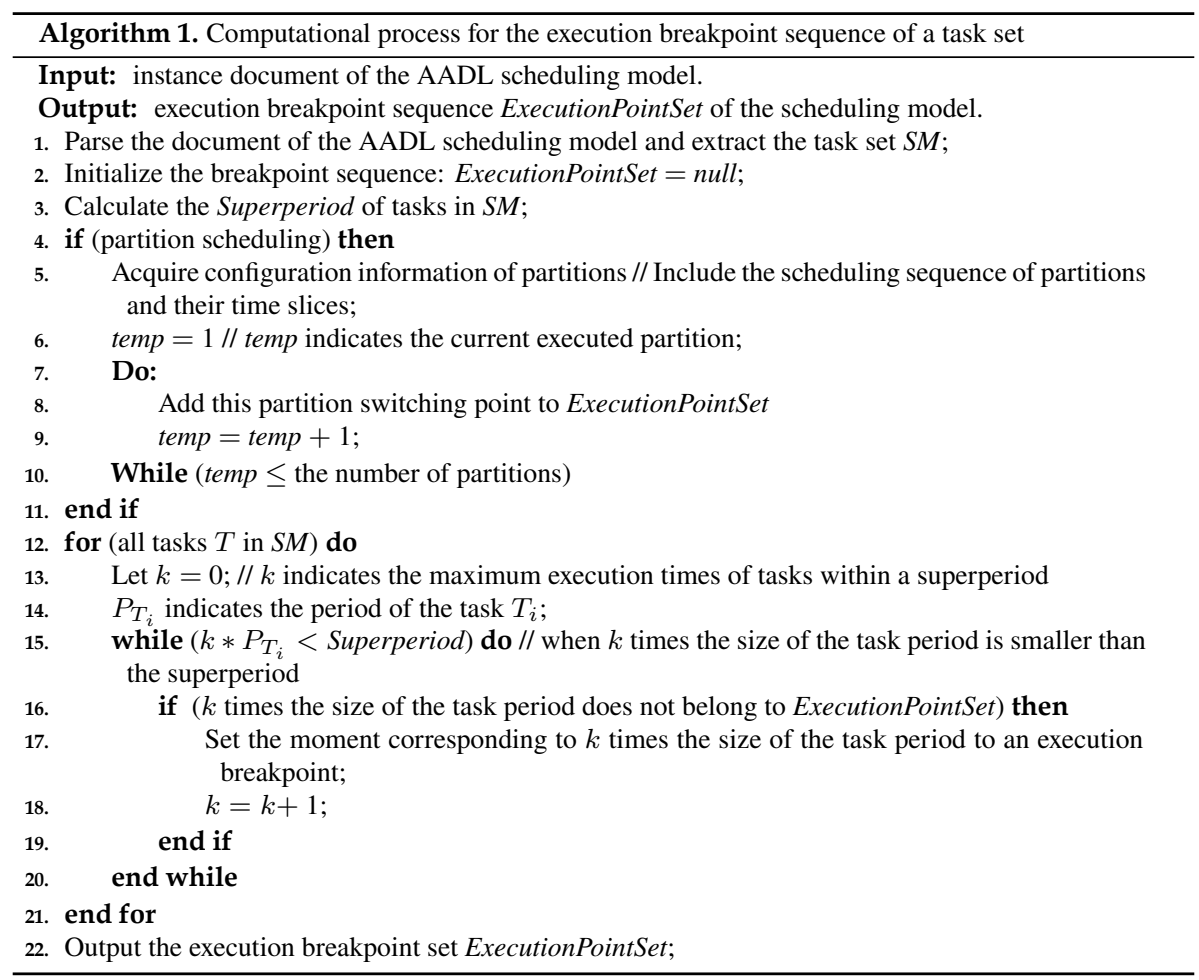

Priority of $T_{2}$ is higher than that of $T_{1}$

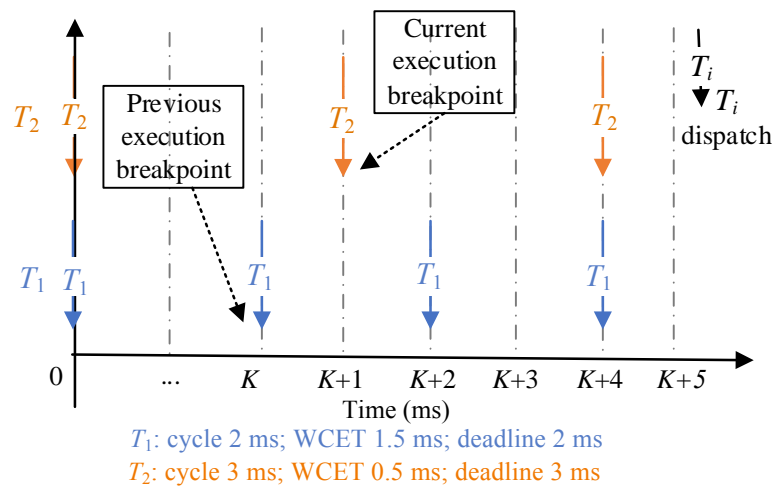

Figure 5 Calculation of preemption points

\subsection{CRPD calculation based on preemption sequences}

In the AADL architecture, a thread component is a basic executable task unit. However, the code implementation of a thread model is not provided directly when AADL models are built. Thus $U C B s$ (the set of UCBs) and ECBs (the set of ECBs) required for task scheduling cannot be obtained according to the model. To support the CRPD calculation of each thread component in AADL models, we need to portray the properties of $U C B s$ and $E C B s$ of preempted tasks in modeling. Thus, we need to define the cache utilization of tasks.

Definition 1. The cache utilization $T_{i}\left(C U_{i}\right)$ of a thread component indicates the ratio of 


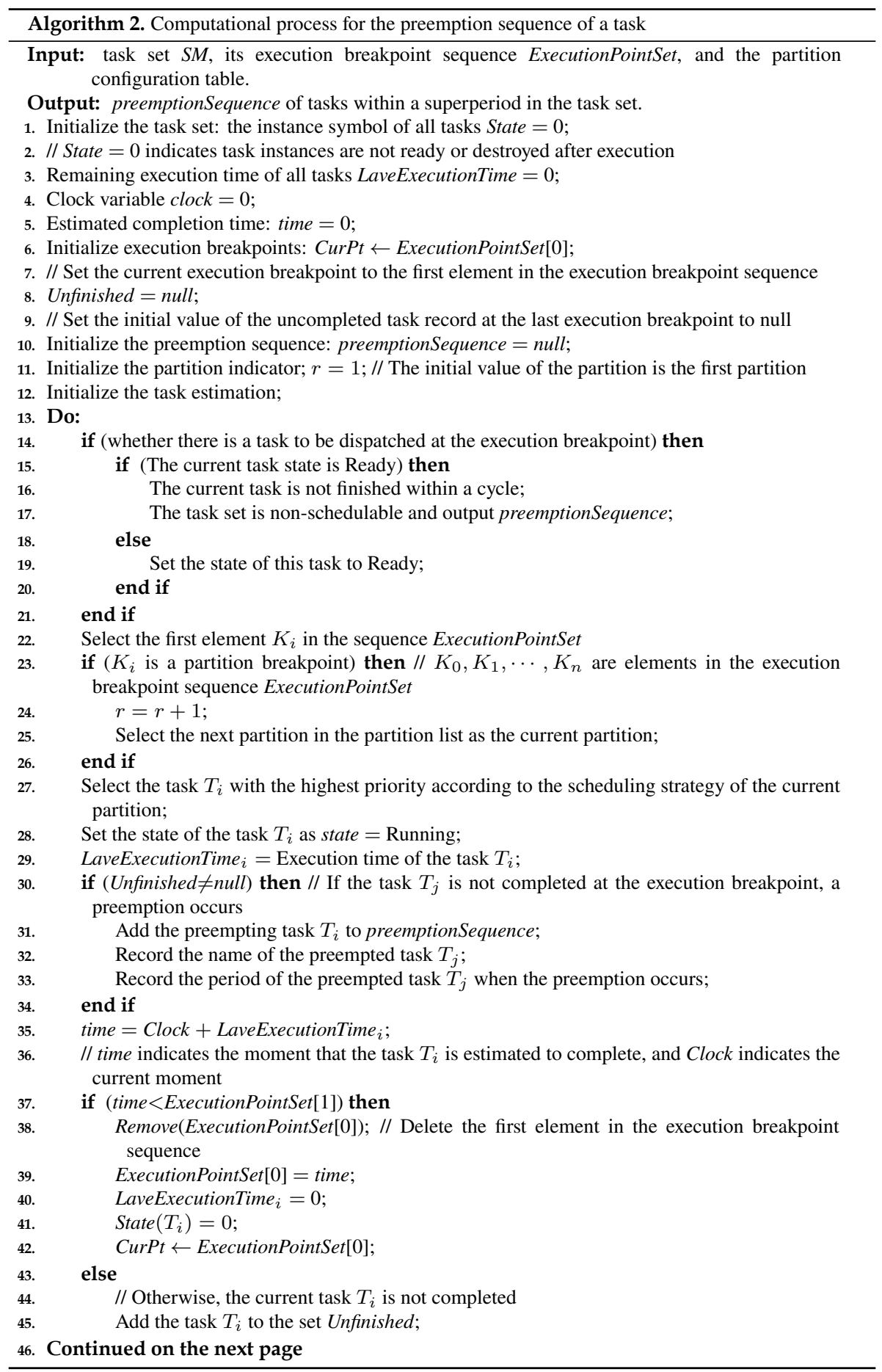




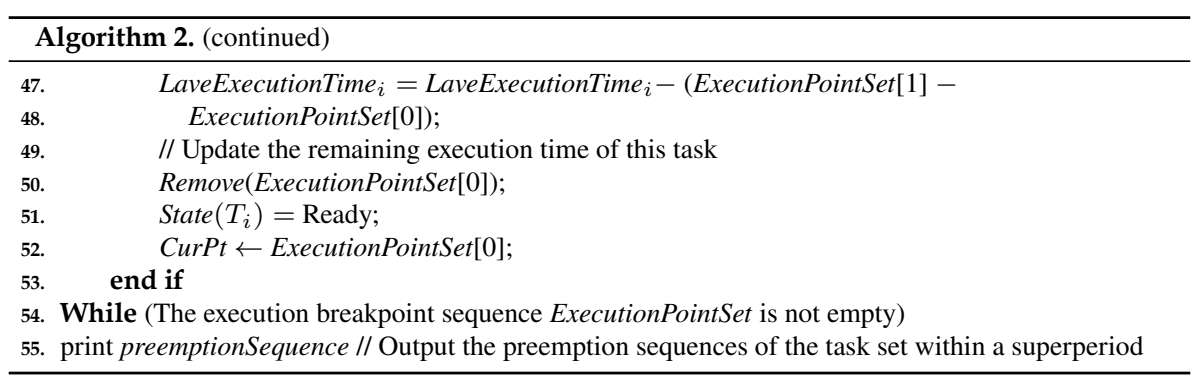

the number of cache blocks occupied by the thread task $T_{i}$ when it runs to the total number of cache blocks. It can be calculated by Equation (4):

$$
C U_{i}=B_{i} / C_{i}
$$

where

(1) $B_{i}$ is the number of cache blocks mapped by the memory space used by the task $T_{i}$ to cache, and its size is determined by the usage amount of ROM and RAM resources of the thread task $T_{i}$ in the AADL architecture model;

(2) $C_{i}$ is the total number of cache blocks.

Thus, the sum of cache utilizations of all threads in a model is called the cache utilization of a system. If the AADL model of a system includes multiple thread components $S=$ $\left\{T_{1}, T_{2}, \cdots, T_{n}\right\}$, the cache utilization of the system can be obtained by Equation (5):

$$
C U=\sum_{i=1}^{n} C U_{i}
$$

When multiple threads use a cache block at the same time, the cache utilization of the system is bigger than 1 .

In AADL, the memory component relies on Memory_Size to describe its storage capacity. The properties and descriptions of the memory component are shown in Table 4.

Table 4 Properties of AADL memory component

\begin{tabular}{cl}
\hline \multicolumn{1}{c}{ Property } & Meaning \\
\hline Source_Code_Size & $\begin{array}{l}\text { Static code and read-only programs generated by compiling and linking programs, } \\
\text { declared in software components, systems, processors, and peripheral components }\end{array}$ \\
Source_Data_Size & $\begin{array}{l}\text { Readable and writable data generated by compiling and linking programs in the } \\
\text { original text, declared in software components, systems, processors, and } \\
\text { peripheral components } \\
\text { The maximum amount of stack space required by processors, peripheral drivers, } \\
\text { threads, and subprograms } \\
\text { The size of heap resources required by threads and subprograms, which is an } \\
\text { interval value }\end{array}$ \\
\hline
\end{tabular}

In storage resource models, the storage resources are categorized as RAM and ROM. AADL classifies the property Source_Code_Size of the memory component as ROM, and the properties Source_Data_Size,Source_Stack_Size, and Source_Heap_Size as RAM. We can also define the properties RomBudget and RamBudget of the memory component, which indicate the estimated ROM and RAM capacities of the component. The properties RomActual and RamActual are adopted to define the actual ROM and RAM capacities used by the memory component. Thus, the number of cache blocks $B_{i}$ mapped to cache by a task is calculated as shown in Equation (6):

$$
B_{i}=\left[\operatorname{ROM}\left(T_{i}\right)+R A M\left(T_{i}\right) / \text { Line_Size }\right]
$$


where (1) $R O M\left(T_{i}\right)$ is the quantity of ROM resources occupied by the task $T_{i}$; (2) $R A M\left(T_{i}\right)$ is the number of RAM resources occupied by the task $T_{i}$; (3) Line_Size is the number of cache blocks, which is determined by the property Line_Size of the AADL model.

According to the cache utilization, ECBs of tasks are generated in a uniformly distributed mode in the cache. The generation of UCBs depends on ECBs. UCBs of each task can be obtained by multiplying the $E C B s$ by a reuse factor $\rho$. Thus, $U C B s$ are a subset of $E C B s$, and the rank of the set satisfies $|U C B s|=\rho|E C B s|$, where $\rho$ is the reuse ratio of cache blocks in the execution of tasks and it is affected by many factors. For example, $\rho$ will be larger when there are loops in the program execution flow. The program locality of data processing threads is not as evident as that of instruction control threads, so the reuse factor of control threads is higher than that of data processing threads. In the model design, $\rho$ is a random variable within the range of $[0,1]$ and follows a uniform distribution ${ }^{[20]}$.

Algorithm 2 is used to calculate preemption sequences of the task set within a superperiod. Combining the preempted moment of the task $T_{i}$ and the cycle of $T_{i}$, the preemption sequence of the task $T_{i}$ in one execution cycle can be obtained. By traversing this sequence, we can obtain the set of preempted cache blocks $C R P D_{i, k}^{j}$ for each preempted task. The union of all $C R P D_{i, k}^{j}$ sets is the total number of possibly accessed cache blocks due to preemption in the execution process of $T_{i}$. The intersection of it and the set of useful cache blocks $C R P D_{i, k}^{j}$ of the preempted task $T_{i}$ is the set of cache blocks that are replaced out. After the task $T_{i}$ is preempted by the task $T_{n}$ in the $k$ th subcycle $(k=1,2, \cdots, m)$, the $C R P D_{i, k}^{j}$ based on the $j$ th preemption sequence can be calculated by Equation (7):

$$
C R P D_{i, k}^{j}=\text { CacheMissTime } \times\left|U C B s_{T_{i}} \cap\left(\bigcup_{T_{n} \in \text { preemptingSequence }\left(T_{i}\right)} \operatorname{ECBs}_{T_{n}}\right)\right|
$$

where preemptingSequence $\left(T_{i}\right)$ indicates the execution sequence of high-priority tasks in the period from the task $T_{i}$ being preempted to resuming execution.

Equation (7) is used to calculate the preemption cost of a preempted task under a preemption sequence, but we need to calculate the preemption cost of the preempted task in a task cycle with multiple preemptions. Since there may be many preemption sequences in the task cycle of a preempted task, the $C R P D$ of the preempted task in one cycle is the sum of the CRPD calculated for all preemption sequences. Thus, $C R P D_{i, k}$ of a preempted task $T_{i}$ in one cycle can be calculated by Equation (8):

$$
C R P D_{i, k}=\sum_{j=1}^{N} C R P D_{i, k}^{j}
$$

Further, the maximum $C R P D_{i}$ of the task $T_{i}$ within a superperiod can be calculated by Equation (9):

$$
C R P D_{i}=\max \left\{C R P D_{i, 1}, C R P D_{i, 2}, \cdots, C R P D_{i, k}\right\}
$$

\subsection{Calculation of WCET and schedulability determination of task set}

The WCET of the task $T_{i}$ is denoted as $W C E T_{i}^{\text {new }}$, and the WCET of the task under the constraint of CRPD can be calculated by Equation (10):

$$
W C E T_{i}^{\text {new }}=W C E T_{i}^{\text {old }}+C R P D_{i}
$$

where 
(1) $W C E T_{i}^{\text {old }}$ is the execution time of $T_{i}$ in the AADL architecture model;

(2) $C R P D_{i}$ is the maximum CRPD of the task $T_{i}$ within a superperiod.

In AADL architecture models, the execution time of a task is confirmed as follows: The Execution_Time property of the thread component in an architecture model confirms the range of the execution time of a task, which indicates the Best-Case Execution Time (BCET) and WCET of the thread. The WCET calculated by Equation (10) is based on the CRPD of each task calculated by preemption sequences generated in the task set within a superperiod. However, different scheduling sequences generate various preemption points. After the WCET of tasks is modified, different scheduling sequences are generated when the task set is reexecuted. Therefore, when analyzing the system schedulability based on preemption cost, we should update WCET continuously until it no longer changes, which indicates that the WCET calculation of the task is completed.

To analyze the real-time performance of a system, we introduce the Worst-Case Response Time (WCRT), which is a key problem in studying real-time scheduling. The basic principle of schedulability determination is to judge whether the WCRT of a task satisfies the deadline constraint. If the WCRT of a task satisfies the deadline constraint, then the task is schedulable.

Scheduling strategies of a system directly influence WCRT of task execution as the method of calculating WCRT varies under different scheduling strategies. For example, when a fixedpriority scheduling strategy is used in an intra-partition scheduling system, the WCRT of the task $T_{i}$ is determined by the execution time $I$ of $T_{i}$ and the interference $I$ of high-priority tasks to $T_{i}$. The interference $I$ is calculated as shown in Equation (11):

$$
I=\sum_{\forall T_{j} \in h p\left(T_{i}\right)} n \times W C E T_{j}^{\text {new }}
$$

where

(1) $h p\left(T_{i}\right)$ is the set of tasks in the scheduling task set, whose priority is higher than that of the task $T_{i}$;

(2) $W C E T_{j}^{\text {new }}$ is the WCET of the task $T_{j}$;

(3) $n$ is the upper bound on the number of times that high-priority tasks are dispatched during the response time of the task $T_{i}$, and it is calculated as shown in Equation (12):

$$
n=\left[\frac{W C R T_{i}}{P_{j}}\right]
$$

where

(1) $W C R T_{i}$ is the WCRT of the task $T_{i}$;

(2) $P_{j}$ is the cycle of the task $T_{j}$.

In a fixed-priority preemptive system, $W C R T_{i}$ of tasks can be calculated by Equation (13):

$$
W C R T_{i}=W C E T_{i}^{\mathrm{new}}+\sum_{\forall j \in h p(i)}\left[\frac{W C R T_{i}}{P_{j}}\right] \times W C E T_{j}^{\text {new }}
$$

To determine the schedulability of a task set, we need to determine whether the response time of tasks in the task set in each cycle exceeds their deadlines within a superperiod. Thus, a property of remaining execution time is added to the thread component model of AADL. When the next execution breakpoint arrives and the task being scheduled is dispatched at the next execution breakpoint, we determine whether the task can be completed when the next execution breakpoint arrives according to the remaining execution time of the task. If the remaining 
execution time of the task being executed is less than the difference between the next execution breakpoint and the current moment, the task can be completed; otherwise, the task is not finished before the next task is dispatched, and the task set is non-schedulable.

The schedulability of task sets in AADL architecture can be determined by calculating the WCRT of each task in light of preemption sequences and comparing the WCRT of each component task with the deadline of the component. Specifically, AADL architecture is schedulable when and only when the WCRT of any component task in the architecture is less than its deadline. The WCET of a task is calculated as shown in Algorithm 3. Algorithm 2 is called in Algorithm 3, and the WCET of each task is calculated. The relationship between the number $n$ of tasks in the task set and the number $k$ of breakpoints in the execution breakpoint sequence satisfies $n \leq k$. Thus, the time complexity of Algorithm 3 is $\mathrm{O}(k)$.

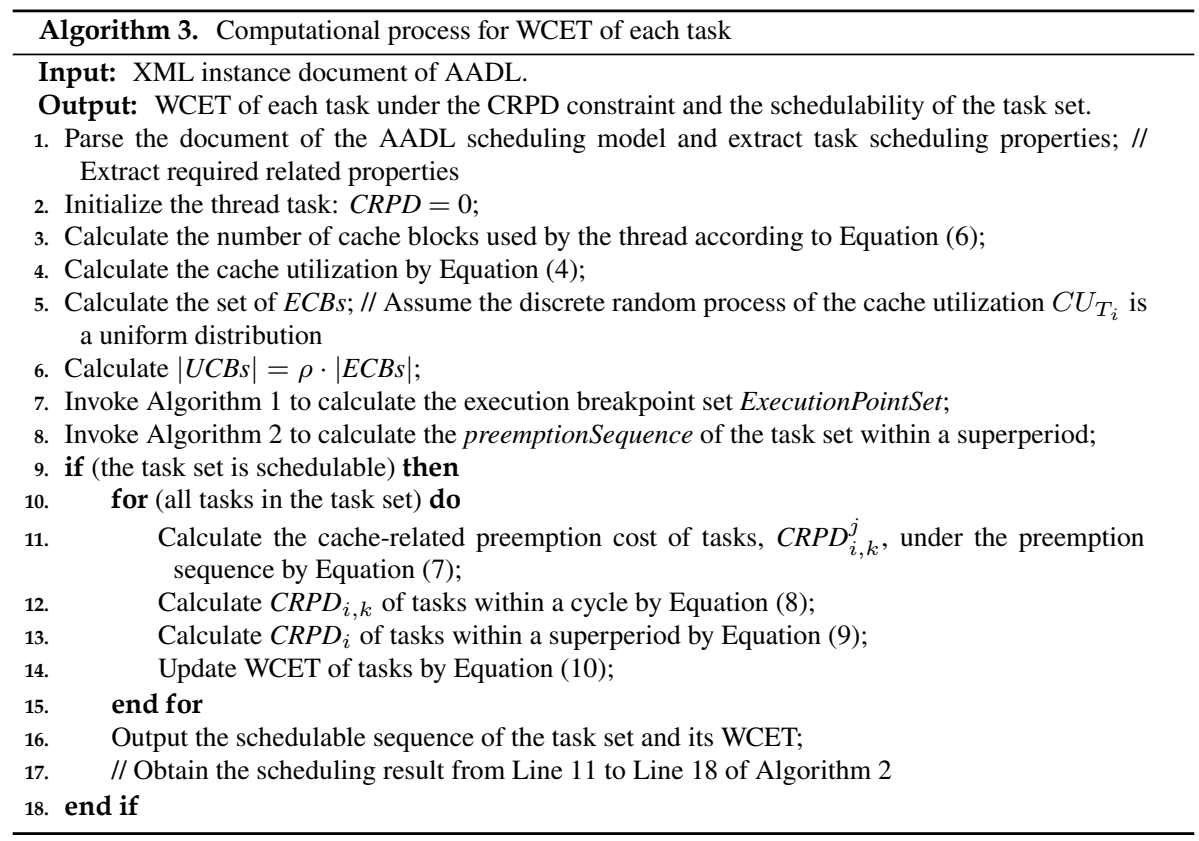

\section{Toolkit Implementation and Case Analysis}

Based on the modeling and analysis theory above, we develop a prototype system of a schedulability analysis toolkit for AADL models by the plug-in development technology, Eclipse, and the system architecture is shown in Figure 6. This toolkit supports AADL architecture modeling, storage resource modeling, and model schedulability analysis of multi-partition and multi-task information systems on the partitioned operating platform ARINC653.

\subsection{Architecture modeling of intelligent information system}

To verify the correctness of theoretical results and the practicality of our prototype tool, we carry out experiments with the intelligent aviation information system as an example. In the intelligent aviation information system, four computational nodes are connected by a double-ring high-speed fiber network to form a highly reliable avionics system. The AADL architecture model of one computational node, the GPM_A subsystem, is shown in Figure 7. 

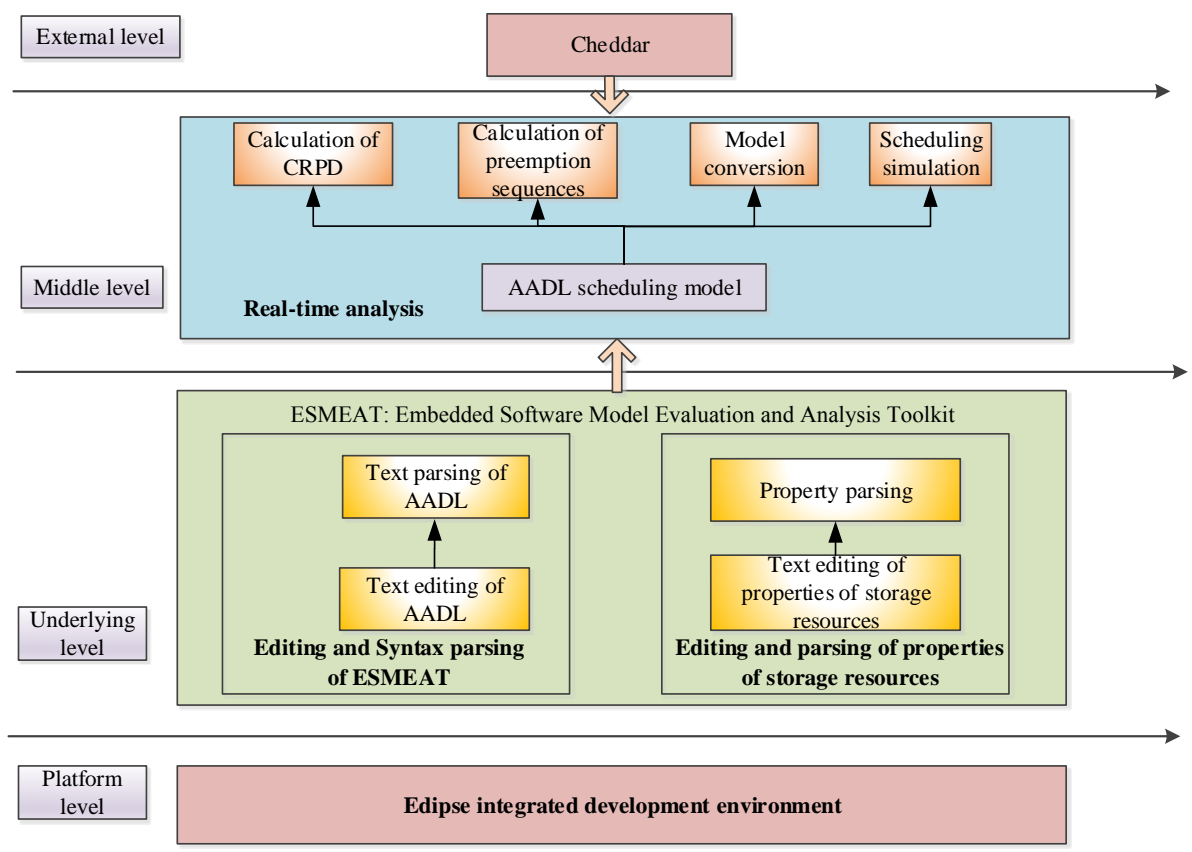

Figure 6 Architecture of schedulability analysis software toolkit

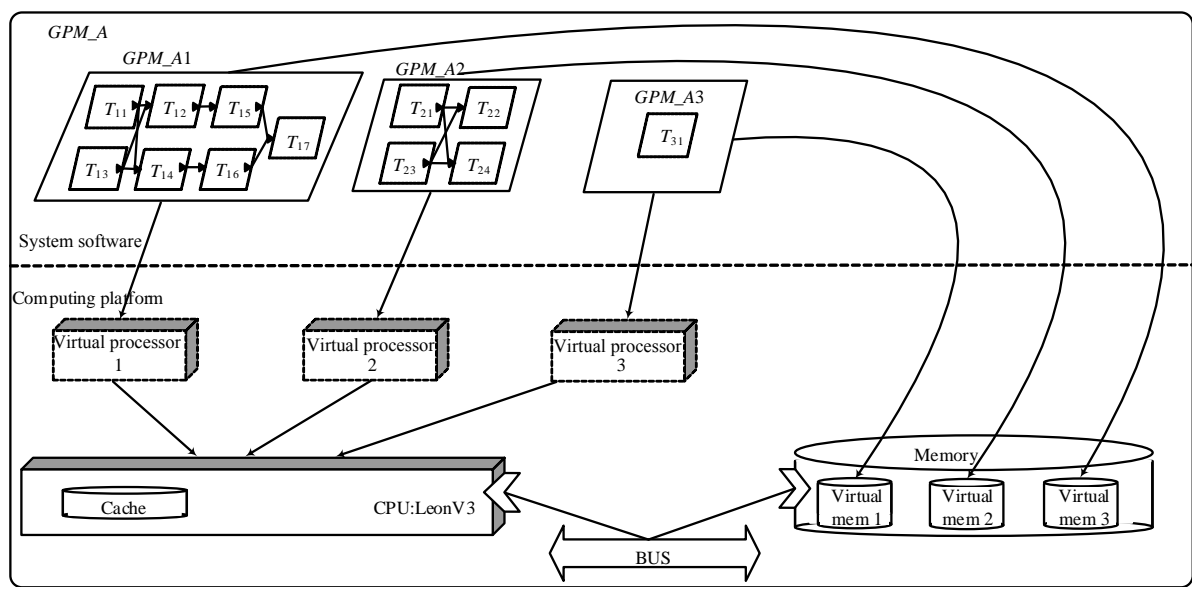

Figure 7 AADL architecture model of a single processor system

Table 5 lists the partition information of $G P M \_A$ and the set of tasks in each partition. The scheduling-related properties of each partition are shown in Table 6 , and the values of scheduling properties of threads in each partition are shown in Table 7. GPM_A1 includes thread components $T_{11}-T_{17}$, which are bound to the virtual processor. It shares the processor component LeonV3 and the memory component with the other two partitions.

\subsection{Experimental analysis of intra-partition scheduling}

In the $G P M \_A$ model of the intelligent aviation information system, the cache capacity of the partition GPM_A1 is $200 \mathrm{~KB}$, and the capacity of a cache block is 32 bytes. The main frequency of the processor Leon is $250 \mathrm{MHz}$, and one cache miss takes $40 \mathrm{~ns}$. The experiment 
adopts the scheduling strategy RMS. The superperiod of the task set in the partition GPM_A1 is $13,200 \mathrm{~ms}$. All tasks are completed at $13,188 \mathrm{~ms}$, and the deadline constraint of each task is satisfied. Thus, the subsystem partition $G P M \_A 1$ is schedulable under the CRPD constraint. As shown in Figure 8, there is no difference in the WCET of the thread $T_{11}$ with and without considering the CRPD constraint after the system executes 20 iterations. This is because $T_{11}$ has the highest priority, and its preemption cost is 0 . However, the differences in the cache-related preemption cost of threads $T_{16}$ and $T_{17}$ with and without considering CRPD are significant as they are preempted several times within a superperiod and thus have a bigger CRPD.

Table 5 Components inside the three partitions of GPM_A

\begin{tabular}{ccc}
\hline Partition & Thread & Meaning of thread \\
\hline & $T_{11}$ & Data collection task \\
& $T_{12}$ & Processing task of drivers' instructions \\
GPM_A1 & $T_{13}$ & Fault warning task \\
& $T_{14}$ & Data output task \\
& $T_{15}$ & Processing task 1 of flight parameters \\
& $T_{16}$ & State machine task 1 \\
& $T_{17}$ & Error handling task 1 \\
\hline & $T_{21}$ & Recoding task 2 of flight parameters \\
GPM_A2 & $T_{22}$ & Error handling task 2 \\
& $T_{23}$ & Message dispatch task \\
& $T_{24}$ & State machine task 2 \\
\hline$G P M_{-} A 3$ & $T_{31}$ & Continuous BIT task \\
\hline
\end{tabular}

Table 6 Length of time slice and intra-partition scheduling strategy of each GPM_A partition

\begin{tabular}{ccc}
\hline Partition & Time slice of partition $(\mathrm{ms})$ & Scheduling strategy of partition \\
\hline$G P M \_A 1$ & 8 & RMS \\
$G P M \_A 2$ & 7 & EDF \\
$G P M \_A 3$ & 3 & RMS \\
\hline
\end{tabular}

Table 7 Properties of tasks related to scheduling within each partition of GPM_A

\begin{tabular}{|c|c|c|c|c|c|}
\hline Task & Partition & $\begin{array}{c}\text { Usage amount of storage } \\
\text { resource }(\mathrm{KB})\end{array}$ & Execution time (ms) & Cycle (ms) & Deadline (ms) \\
\hline$T_{11}$ & GPM_A1 & 400 & 3 & 15 & 15 \\
\hline$T_{12}$ & $G P M \_A 1$ & 440 & 5 & 22 & 22 \\
\hline$T_{13}$ & $G P M \_A 1$ & 300 & 5 & 60 & 60 \\
\hline$T_{14}$ & $G P M \_A 1$ & 240 & 3 & 60 & 60 \\
\hline$T_{15}$ & $G P M \_A 1$ & 330 & 4 & 75 & 75 \\
\hline$T_{16}$ & $G P M \_A 1$ & 320 & 4 & 80 & 80 \\
\hline$T_{17}$ & GPM_A1 & 260 & 5 & 100 & 100 \\
\hline$T_{21}$ & $G P M \_A 2$ & 280 & 3 & 40 & 40 \\
\hline$T_{22}$ & GPM_A2 & 300 & 4 & 80 & 80 \\
\hline$T_{23}$ & $G P M \_A 2$ & 200 & 3 & 200 & 200 \\
\hline$T_{24}$ & $G P M \_A 2$ & 330 & 3 & 100 & 100 \\
\hline$T_{31}$ & $G P M \_A 3$ & 14 & 3 & 80 & 80 \\
\hline
\end{tabular}

Similarly, Figure 9 provides the comparison of CRPD results calculated by the scheduling algorithm based on preemption sequences with those of the UCB-Union and ECB-Union methods. The CRPD calculated based on preemption sequences is more accurate than that calculated by UCB-Union and ECB-Union. The experimental results show that the CRPD calculated by the scheduling method based on preemption sequences decreases by $73 \mu \mathrm{s}$ at the maximum compared with that of the UCB-Union method, and by $23 \mu \mathrm{s}$ at the maximum compared with that of the ECB-Union method. When the intra-partition scheduling method is adopted, the cache-related preemption cost of a task is negatively correlated with the priority of the task in general. 


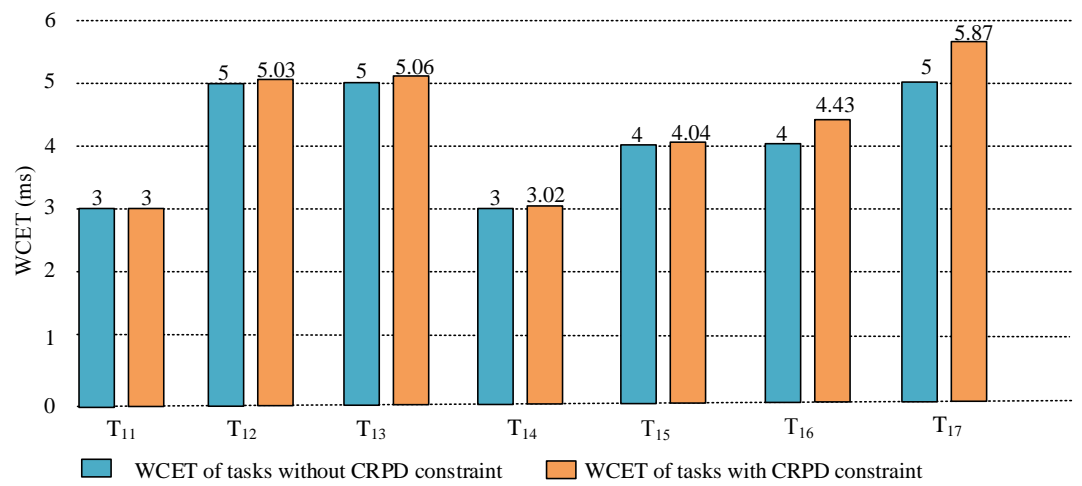

Figure 8 Comparison of WCET calculated with or without CRPD constraint of tasks within partition GPM_A1

\subsection{Experimental analysis of partition scheduling}

When $G P M \_A$ in the intelligent aviation information system follows partition scheduling, the cache capacity is $500 \mathrm{~KB}$, and the capacity of a cache block is 32 bytes. The main frequency of the processor is $250 \mathrm{MHz}$, and one cache miss takes $40 \mathrm{~ns}$. The partition scheduling information is shown in Table 7, and a superperiod of tasks is $13,200 \mathrm{~ms}$. When tasks are executed according to the execution sequence for $60 \mathrm{~ms}$, the task $T_{14}$ is preempted by the task $T_{11}$. However, the deadline of $T_{14}$ has been reached, so it is non-schedulable. Thus, the task set is non-schedulable. The specific scheduling sequence is $T_{11} \rightarrow T_{12} \rightarrow T_{21} \rightarrow T_{22} \rightarrow T_{31} \rightarrow T_{12} \rightarrow T_{11} \rightarrow$ $T_{12} \rightarrow T_{13} \rightarrow T_{22} \rightarrow T_{24} \rightarrow T_{12} \rightarrow T_{11} \rightarrow T_{12} \rightarrow T_{23} \rightarrow T_{12} \rightarrow T_{12} \rightarrow T_{11} \rightarrow T_{21} \rightarrow$ $T_{12} \rightarrow T_{14}$ (the task $T_{14}$ fails to complete execution before the deadline).

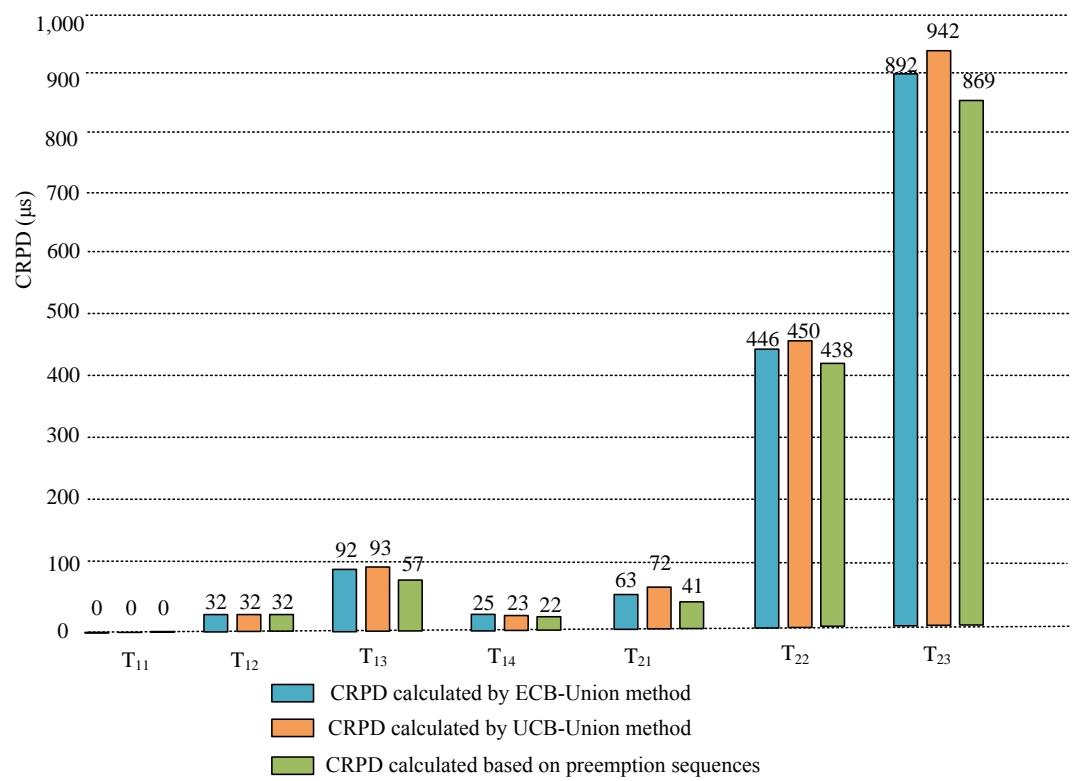

Figure 9 Comparison of CRPD calculated with different algorithms

As the cycles of tasks in the partition $G P M_{-} A 1$ are too short, under time slice round robin scheduling, the short-cycle tasks in GPM_A1 keep preempting other tasks, which causes the 
long-cycle tasks to exceed their deadlines and become non-schedulable. During the system architecture design stage, we need to optimize the system design by adjusting the partition configuration of GPM_A1 and its task scheduling properties. The adjusted scheduling properties of the system are shown in Table 8 .

Table 8 Adjusted task scheduling properties in each partition of GPM_A with CRPD constraint

\begin{tabular}{cccccc}
\hline Task & Partition & $\begin{array}{c}\text { Usage amount of storage } \\
\text { resource }(\mathrm{KB})\end{array}$ & $\begin{array}{c}\text { Execution time }(\mathrm{ms}) \\
\text { Cycle (ms) }\end{array}$ & Deadline (ms) \\
\hline$T_{11}$ & $G P M \_A 1$ & 400 & 3 & 40 & 40 \\
$T_{12}$ & $G P M \_A 1$ & 440 & 5 & 80 & 80 \\
$T_{13}$ & $G P M \_A 1$ & 300 & 5 & 80 & 80 \\
$T_{14}$ & $G P M \_A 1$ & 240 & 3 & 100 & 100 \\
$T_{15}$ & $G P M \_A 1$ & 330 & 4 & 100 & 100 \\
$T_{16}$ & $G P M \_A 1$ & 320 & 4 & 80 & 80 \\
$T_{17}$ & $G P M \_A 1$ & 260 & 5 & 100 & 100 \\
$T_{21}$ & $G P M \_A 2$ & 280 & 3 & 40 & 40 \\
$T_{22}$ & $G P M \_A 2$ & 300 & 4 & 80 & 80 \\
$T_{23}$ & $G P M \_A 2$ & 200 & 3 & 200 & 200 \\
$T_{24}$ & $G P M \_A 2$ & 330 & 3 & 100 & 100 \\
$T_{31}$ & $G P M \_A 3$ & 14 & 3 & 80 & 80 \\
\hline
\end{tabular}

The superperiod of tasks in three partitions of the optimized system is $400 \mathrm{~ms}$, and the three partitions adopt different scheduling strategies. All tasks are completed at $381 \mathrm{~ms}$ and satisfy the deadline constraints. Thus, they are schedulable under the constraint of CRPD. When the three partitions in the optimized system adopt the same scheduling strategy, the WCET of the system with and without CRPD constraint is compared, as shown in Figure 10.

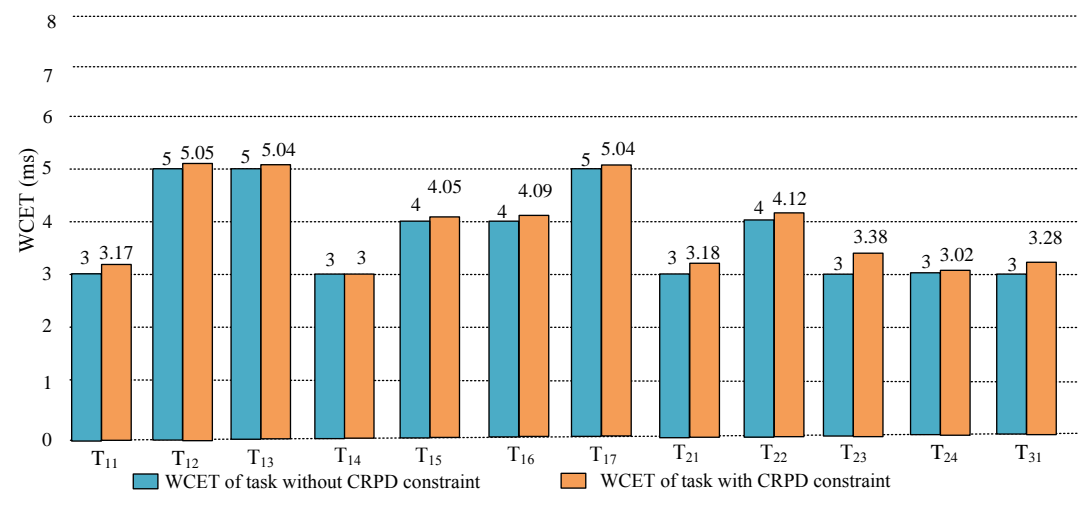

Figure 10 WCET calculated with or without CRPD of all tasks inside each partition of GPM_A

In system architecture, the cache capacity influences the frequency of cache misses in the scheduling process of systems and also affects the system schedulability. When the cache miss moments remain unchanged, the CRPD of tasks under different cache capacities is shown in Table 9, and the CRPD of each task varying with the cache capacity is shown in Figure 11. As the cache capacity increases, CRPD reduces.

\section{Conclusion and Prospect}

This paper studies the schedulability of AADL models under the CRPD constraint for complex embedded systems. We extend the properties of AADL so that it can build the cache model. On this basis, we propose the schedulability analysis method for AADL models under the CRPD constraint and calculate CRPD of preempted tasks by calculating preemption sequences 
of task sets under a specific scheduling strategy. Our method considers the impact of preemption between tasks on the system schedulability on the system architecture level. In addition, it is effective with good generality and ensures the security of WCET under the CRPD constraint. In this paper, we design and develop a prototype of the schedulability analysis toolkit for the AADL architecture model with storage resource constraints, and conduct experiments with the intelligent aviation information system as an example. The experimental results show that the CRPD results obtained by the calculation method based on preemption sequences are more accurate than those of the UCB-Union and ECB-Union methods. When the task set of the system is not schedulable, we analyze the reason for non-schedulability and provide reasonable optimization suggestions to make the optimized system schedulable. At last, we evaluate the impact of cache capacity on CRPD of tasks for the reference of system designers.

Table 9 CRPD calculated with different cache sizes equipped for each task ( $\mu \mathrm{s})$

\begin{tabular}{cccccccc}
\hline \multirow{2}{*}{ Task } & \multicolumn{7}{c}{ Cache capacity } \\
\cline { 2 - 8 } & $500 \mathrm{~KB}$ & $750 \mathrm{~KB}$ & $1 \mathrm{MB}$ & $1.25 \mathrm{MB}$ & $1.5 \mathrm{MB}$ & $1.75 \mathrm{MB}$ & $2 \mathrm{MB}$ \\
\hline$T_{11}$ & 156 & 118 & 94 & 54 & 48 & 48 & 35 \\
$T_{12}$ & 51 & 53 & 38 & 32 & $22 \mathrm{~s}$ & 24 & 17 \\
$T_{13}$ & 43 & 34 & 27 & 21 & 13 & 14 & 12 \\
$T_{14}$ & 6 & 6 & 4 & 5 & 2 & 2 & 1 \\
$T_{15}$ & 51 & 37 & 42 & 33 & 27 & 25 & 25 \\
$T_{16}$ & 85 & 54 & 34 & 32 & 19 & 18 & 18 \\
$T_{17}$ & 41 & 45 & 31 & 28 & 22 & 22 & 20 \\
$T_{21}$ & 183 & 150 & 87 & 52 & 43 & 44 & 30 \\
$T_{22}$ & 121 & 84 & 51 & 28 & 21 & 24 & 26 \\
$T_{23}$ & 383 & 314 & 256 & 231 & 161 & 160 & 153 \\
$T_{24}$ & 24 & 19 & 18 & 13 & 4 & 4 & 3 \\
$T_{31}$ & 281 & 134 & 89 & 49 & 42 & 43 & 32 \\
\hline
\end{tabular}

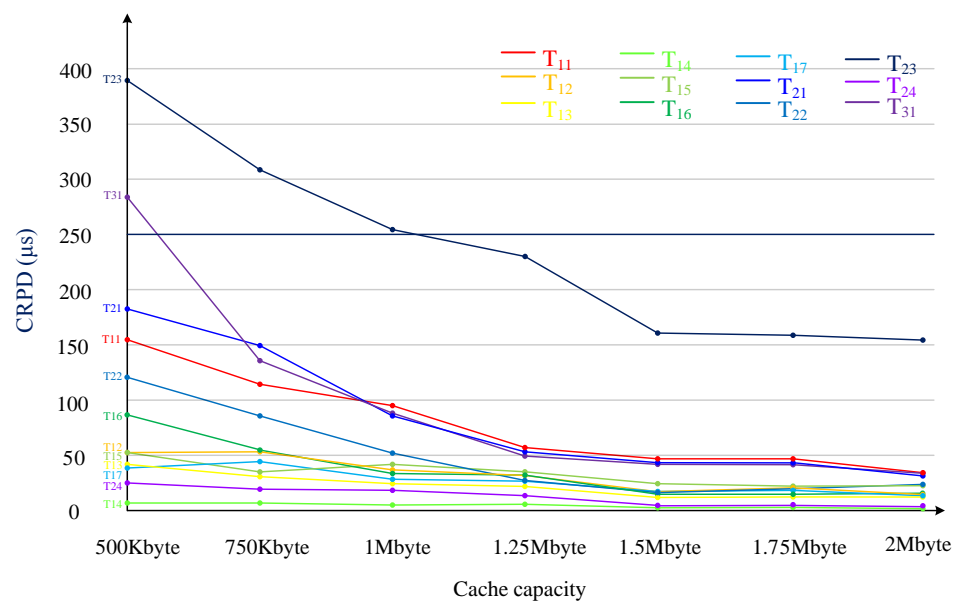

Figure 11 Impact of cache size on CRPD of tasks

The CRPD calculation method based on preemption sequences calculates the CRPD generated by preemption between tasks after obtaining preemption sequences under a specific scheduling algorithm, and it analyzes the scheduling behavior of the system when preemptions occur by introducing WCET. However, many factors are affecting the schedulability of tasks, such as the differences in the methods of calculating preemption sequences of tasks under different scheduling strategies and the synchronous relationship between tasks in models. Thus, 
how to obtain preemption sequences considering scheduling strategies and the synchronous relationship is a problem urgently to be solved.

\section{References}

[1] Liu YF, Zhang LC. Worst-Case execution time analysis for real-time systems. Ji Suan Ji Ying Yong Yan Jiu/Application Research of Computers, 2005, 22(11): 16-18.

[2] Zhou GC, Guo BL, Gao X, et al. Fast estimation of WCET based on distribution function. Ji Suan Ji Ke Xue/Computer Science, 2016, 43: 157-161.

[3] SAE. Architecture analysis and design language (AADL) AS-5506A [Technical Report]. The Engineering Society for Advancing Mobility and Sea Air and Space, Aerospace Infonnation Report. Version 2.0. 2009.

[4] Yang ZB, Pi L, Hu K, et al. AADL: An architecture design and analysis language for complex embedded real-time systems. Ruan Jian Xue Bao/Journal of Software, 2010, 21(5): 899-915. http: //www.jos.org.cn/1000-9825/3700.htm.

[5] Zhang J. Research on the earliest deadline priority real-time scheduling algorithm [Ph.D. Thesis]. Wuhan: Huazhong University of Science and Technology, 2009 (in Chinese with English abstract).

[6] Clarke D, Lee I, Xie HL. VERSA: A tool for the specification and analysis of resource-bound real-time systems. Nonlinear Dynamics, 2016, 87(1): 1-7.

[7] Clavel M, Duran F, Eker S, et al. Maude Manuel. Version 2.1. Menlo Park: SRI Int'1, 2004. http: $/ /$ maude.cs.uiuc.edu.

[8] Jerad C, Barkaoui K, Grissa-Touzi A. On the use of real-time Maude for architecture description and verification: A case study. Proc. of the Visions of Computer Science-bcs Int'l Academic Conf. DBLP, 2015.

[9] Hu K, Duan ZB, Wang JY, et al. Template-Based AADL automatic code generation. Frontiers of Computer Science in China, 2019, 13(4): 698-714.

[10] Singhoff F, Legrand J, Nana L, et al. Scheduling and memory requirements analysis with AADL. Proc. of the ACM Sigada Int'l Conf. on Ada: The Engineering of Correct \& Reliable Software for Real-time \& Distributed Systems Using Ada \& Related Technologies. ACM, 2005. 1-10.

[11] Li ZS. Research on verification method of AADL behavior model based on UPPAAL. Ji Suan Ji Ke Xue/Computer Science, 2012, 39(2): 159-161, 169.

[12] Ma BZ. The worst response time analysis considering the impact of cache replacement [Ph.D. Thesis]. Changsha: Hunan University, 2013 (in Chinese with English abstract).

[13] Tran HN, Singhoff F. Instruction cache in hard real-time systems: Modeling and integration in scheduling analysistools with AADL. Proc. of the Embedded and Ubiquitous Computing. IEEE, 2014. 104-111.

[14] Lee CG, Hahn H, Seo YM. Analysis of cache-related preemption delay in fixed-priority preemptive scheduling. Proc. of the IEEE Computer Society. 1998. 700-713.

[15] Delange J, Pautet L, Plantec A, et al. Validate, simulate, and implement ARINC653 systems using the AADL. ACM SIGAda Ada Letters, 2009, 29(3): 31-44.

[16] Dimpsey RT, Iyer RK. Modeling and measuring multiprogramming and system overheads on a sharedmemory multiprocessor: Case study. Journal of Parallel and Distributed Computing, 1991, 12(4): $402-414$.

[17] Tran HN, Singhoff F, Rubini S. Cache-Aware real-time scheduling simulator: Implementation and return of experience. ACM SIGBED Review, 2016, 13(1): 22-28.

[18] Li C, Ding C, Shen K. Quantifying the cost of context switch. Proc. of the Workshop on Experimental Computer Science, Part of ACM FCRC. San Diego: ACM, 2007. 1-4.

[19] Bastoni A, Brandenburg B, Anderson JH. Is semi-partitioned scheduling practical. Proc. of the Euromicro Conf. on Real-time Systems. IEEE Computer Society, 2011. 1-11.

[20] Bini E, Buttazzo GC. Measuring the performance of schedulability tests. Real-time Systems, 2005, 30(1-2): 129-154. 


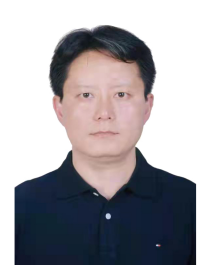

Yin Lu, Ph.D., lecturer, CCF professional member. His research interests include the research on architecture and reliability engineering of embedded systems.

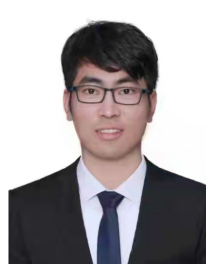

Leqi Xi, master. His research interests include the research on resource scheduling and trusted property analysis of embedded systems.

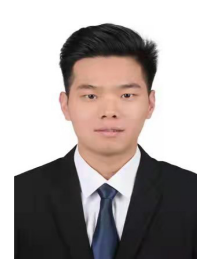

Shudong Qin, master, CCF student member. His research interests include the research on functional property analysis and reliability engineering of embedded systems.

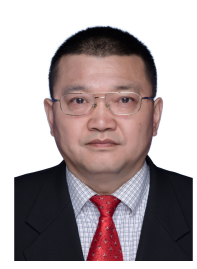

Yunwei Dong, Ph.D., professor, Ph.D. supervisor, $\mathrm{CCF}$ distinguished member. His research interests include the research on design and verification of embedded software, cyberphysical systems, and system modeling and analysis. 